Mon. Not. R. Astron. Soc. 000, 000-000 (0000) Printed 2 March $2022 \quad$ (MN LATEX style file v2.2)

\title{
Was the nineteenth century giant eruption of Eta Carinae a merger event in a triple system?
}

\author{
S.F. Portegies Zwart ${ }^{1}$ and E.P.J. van den Heuvel $^{2,3}$ \\ ${ }^{1}$ Leiden Observatory, Leiden University, PO Box 9513, 2300 RA, Leiden, The Netherlands \\ 2 Astronomical Institute Anton Pannekoek, University of Amsterdam, P.O. Box 94249, 1090 GE, Amsterdam, The Netherlands \\ ${ }^{3}$ Kavli Institute for Theoretical Physics, UCSB, Santa Barbara, CA 93106-4030
}

\begin{abstract}
We discuss the events that led to the giant eruption of Eta Carinae, and find that the mid-nineteenth century (in 1838-1843) giant mass-loss outburst has the characteristics of being produced by the merger event of a massive close binary, triggered by the gravitational interaction with a massive third companion star, which is the current binary companion in the Eta Carinae system. We come to this conclusion by a combination of theoretical arguments supported by computer simulations using the Astrophysical Multipurpose Software Environment. According to this model the $\sim 90 \mathrm{M}_{\odot}$ present primary star of the highly eccentric Eta Carinae binary system is the product of this merger, and its $\sim 30 \mathrm{M}_{\odot}$ companion originally was the third star in the system.

In our model the Homunculus nebula was produced by an extremely enhanced stellar wind, energized by tidal energy dissipation prior to the merger, which enormously boosted the radiation-driven wind mass-loss. The current orbital plane is then aligned with the equatorial plane of the Homunculus, and the symmetric lobes are roughly aligned with the argument of periastron of the current Eta Carina binary. The merger itself then occurred in 1838, which resulted in a massive asymmetric outflow in the equatorial plane of the Homunculus. The 1843 outburst can in our model be attributed to the subsequent encounter when the companion star (once the outer most star in the triple system) plunges through the bloated envelope of the merger product, once when it passed periastron again. We predict that the system has an excess space velocity of order $50 \mathrm{~km} / \mathrm{s}$ in the equatorial plane of the Homunculus. Our triple model gives a viable explanation for the high runaway velocities typically observed in LBVs (Smith \& Tombleson 2015).
\end{abstract}

Key words: stars: individual: Eta Carinae

\section{INTRODUCTION}

Today, Eta Carinae is a binary system, composed of a $\sim 90 \mathrm{M}_{\odot}$ primary star with an estimated age of $\lesssim 1 \mathrm{Myr}$ (Davidson \& Humphreys 1997), although the current copious mass loss rate of $1.6 \times 10^{-3} \mathrm{M}_{\odot} / \mathrm{yr}$ (van Boekel et al. 2003 ), might indicate an older age, possibly on the verge of turning into a Wolf-Rayet star (Smith \& Owocki 2006). The primary is orbited by a $30 \mathrm{M}_{\odot}$ presumably main-sequence star in a $15.46 \mathrm{AU}$ orbit with an eccentricity of about 0.9 (Damineli et al. 1997).

In the mid 19-th century Eta Carinae experienced a giant eruption. Evidence for this is still visible in its surrounding nebula (Humphreys \& Davidson 1999). During this event it outshone any star in the sky except Sirius (de Vaucouleurs \& Eggen 1952). There is no satisfactory scenario that explains the copious mass loss during the 19th century giant eruption, and there is no ready explanation for the at least 2 magnitude bolometric brightening of the object in the $\gtrsim 30$ years before the great eruption (Smith 2013). We propose that both are related, and caused by the eventual merger of a massive close binary, which was triggered by gravitational interaction with a third star in a wide orbit. This third component became Eta Carinae's binary companion, after the merger event. This model has a number of attractive characteristics and interesting consequences for other massive triples to produce objects like Eta Carinae.

Eta Carinae is extraordinary in many respects. The system is often referred to as a 'supernova impostor', and possible candidates that may have met a similar fate include SN1954J Smith et al. (2001), V838 Mon (Munari et al. 2002), SN2009ip (Maza et al. 2009), SN1961v (Kochanek 
et al. 2012), and $\delta$ Scorpius (Miroshnichenko et al. 2013). The comparison with the outburst in SN2009ip (Smith et al. 2011) may be particularly important in understanding the diversity in spectral properties of eruptions in luminous blue variables in general (Foley et al. 2011). The high mass and high velocity of the great eruption of Eta Carinae and the outburst in SN2009ip may then have a similar origin, possibly resembling that of a common envelope event (Mauerhan et al. 2013; Tsebrenko \& Soker 2013; Levesque et al. 2014). These events are referred to as "mergerburst" by Soker \& Kashi (2013). Supernova impostor PTF11iqb was initially classified as a type SN II-N (Parrent et al. 2011) but with weaker pre-supernova mass loss, as seen in SNe II-L and II-P, but was later characterised as an event similar to Eta Carinae (Smith et al. 2015). Similarly, the NGC 300 OT2008-1 event was suggested to be a scaled down version of the same process that drove the great eruption in Eta Carinae (Kashi et al. 2010).

Potential future candidates include HD75821, HD167263 and HD135240 (Eggleton \& Tokovinin 2008, 2010), and the circumstellar nebular R4 has characteristics of having experienced a similar binary merger (Pasquali et al. 2000).

Several of today's parameters of Eta Carinae are well constrained by the observations, but what initiated the great eruption and how the system looked like before this event is actively debated among astronomers. In this paper we aim at constraining the parameters and the events that led to the giant eruption, but we start constraining some of the current observables. For example, to avoid violation of the Eddington limit for Eta Carina's luminosity $\left(L_{\text {bol }}=10^{6.7} \mathrm{~L}_{\odot}\right)$ during its great eruption, its mass must be $\gtrsim 80 \mathrm{M}_{\odot}$ (Humphreys \& Martin 2012). The star has a large wind mass loss rate and enhanced atmospheric $\mathrm{N}$ and He abundances and reduced $\mathrm{C}$ and $\mathrm{O}$ abundances. This indicates that it is already in an advanced stage of core-hydrogen burning and has lost quite a lot of mass by its strong wind and eruptions. This also points to a high initial mass, possibly well in excess of $200 \mathrm{M}_{\odot}$ (Humphreys \& Martin 2012).

We propose here the possibility that a third star in a relatively wide orbit sparked the event, and discuss the physics of the ejection process in this model. Also if our model would finally not be the one that explains all the chanacteristics of Eta Car, still and evolution as discribed and modelled here is expected to happen not rarely in nature.

Hierarchical triples are common among massive stars (Sana et al. 2012), and the orbital parameters required for the proposed scenario to work appear to be rather common. A triple origin of the great eruption has been suggested before by Livio \& Pringle (1998), in which an exchange in a dynamically unstable triple would have initiated the event, and by Kundt \& Hillemanns (2003) who argued that an accretion disk around a tertiary neutron star would have sparked the great eruption.

According to our scenario, Eta Carinae was born a hierarchical triple, in which a tight inner binary star is orbited by a tertiary star in a wide orbit. The inner binary system could have been composed of a $M \gtrsim 90 \mathrm{M}_{\odot}$ primary star with an $m \simeq 10-40 \mathrm{M}_{\odot}$ companion in a rather tight $a_{\text {in }} \simeq 1 \mathrm{AU}$ orbit. By the time of the merger, the primary has lost some $10-20 \mathrm{M}_{\odot}$ in a dense stellar wind. A third companion in a relatively wide orbit drives the inner binary, via the Lidov-Kozai mechanism (Lidov 1962; Kozai 1962) to a state of tidal evolution followed by coalescence.

A strong interaction between two stars has been demonstrated to be able to effectively drive a massive outflow similar to that observed in Eta Carinae (Harpaz \& Soker 2009; Smith 2011). A similar model in which two main-sequence stars coalesce, was presented by Kato (2003). Kato based his idea on the model by (Soker \& Tylenda 2003) for V838 Monocerotis (see also Tylenda \& Soker 2006). Other binary merger models are those for V1309 Scorpius (Tylenda et al. 2011; Nandez et al. 2014) and V4332 Sagittarii (Tylenda et al. 2014). Due to the large distance, imaging of the ejecta in these cases is not feasable, except for V838 Mon which appears to be rather assymetric (Soker \& Tylenda 2003). The lightcurves of some of these potential merger events show characteristics very similar to that of the great eruption in Eta Carniae (Kashi et al. 2010).

The great eruptions in Eta Carina (but also of V838 Mon) appears to have occurred near the pericenter of the current binary system (Damineli 1996; Kashi \& Soker 2010; Smith \& Frew 2011). As we will discuss in $\S 5$ this is consistent with the model we discuss here. In our case the pericenter passage of the tertiary star initiates the merger of the inner binary system, leading to the first great eruption of 1838. The mass loss and associated velocity assymmetry induced upon the binary merger causes the very wide and eccentric outer orbit to shrink to its present size. At the time of the second periastron passage in 1843 we expect the merger product still to have been a quite extended star, such that the new secondary collided with its outer layers, causing the large 1843 eruption. The periastron passage of the outer companion at the time of the merger, and therefore at the time of the great 1843 eruption, is thus a natural consequence of our simulations, independently of the observations.

If certain conditions are met, the resulting binary system will resemble Eta Carinae. In this paper we discuss the conditions required for the scenario to work, and argue that this model may provide a plausible explanation for a number of curious observables for the Eta Carinae system.

We will discuss the evolution of the triple system in three phases A, B and C, which we relate to phases in the observed evolution of Eta Car. The parameters describing the system are indicated with the appropriate superscripts for the conditions upon birth (A), the situation just before Roche-lobe overflow in 1838 (B), and after the merger is completed (C).

\section{ETA CARINAE BEFORE THE GREAT ERUPTION (PHASE A)}

At the age of $0.1-1 \mathrm{Myr}$, the $\sim 90 \mathrm{M}_{\odot}$ and $30 \mathrm{M}_{\odot}$ stars have radii as large as $15-20 R_{\odot}$ and $4-9 R_{\odot}$ respectively. With an orbit separation of $70-400 \mathrm{R}_{\odot}$, these stars will experience strong tidal interactions when the eccentricity grows to $e \gtrsim 0.6$. As a result of such interaction the binary circularizes, the rotation of the two stars synchronizes. The tidal energy that is dissipated in the stellar envelope gives rise to a dramatic stellar wind from the primary star, until the two stars eventaly engage in a common envelope (Webbink 1984; Eggleton 2006). 
A high eccentricity can be induced by a third body via the Lidov-Kozai (Kozai 1962) effect. The rate at which this drives the growth of the eccentricity of the inner orbit can be calculated from secular perturbation theory (Ford et al. 2000). The time scale of the secular growth can be comparable to the orbital period of the inner binary. The growth of the inner eccentricity is particularly rapid when the triple is barely dynamically stable (Mardling 2008; Hamers et al. 2015). It is in this range of parameter space that the strongest interactions are expected, and collisions may occur.

It is important to notice, as we will show in the next section, that the tidal interaction may, already for many decades prior to the merger, induce a much enhanced stellar wind from the primary star, leading to a loss of more than $10 \mathrm{M}_{\odot}$ prior to the merger (see fig. 2). We now will quantitatively study the evolution of the triple-star model.

\subsection{Numerical integration of the triple before the merger leading to a great eruption}

To quantify the orbital evolution of Eta Carina before the great eruption in terms of the triple star model we follow the dynamical, stellar and tidal evolution of the triple system. We adopted the Astronomical Multipurpose Software Environment (AMUSE for short Portegies Zwart et al. 2013) ${ }^{1}$ ) for this integration, by coupling the various components and integrating the coupled system.

Gravity was solved using the Huayno direct $N$-body integrator (Pelupessy et al. 2012). Energy in the dynamical simulations is preserved to better than $1 / 10^{12}$ with respect to the initial energy of the system, which is sufficient for a reliable integration (Portegies Zwart \& Boekholt 2014). Tidal evolution was incorporated for the two inner stars, but not for the tertiary, using the tidal dissipation routine in AMUSE, which is based on Eggleton et al. (1998, see also Hansen (2010)). For stellar evolution we adopted the MESA Henyey stellar evolution code (Paxton et al. 2011). All stars are assumed to be born with an angular frequency of $\Omega=2.6 \times 10^{-6} \mathrm{~s}^{-1}$. Each time step we calculate the amount of energy dissipated in the tidal interaction.

In the AMUSE script each of the three numerical solvers for stellar evolution, tidal evolution and gravitational dynamics is run subsequently, switching between them after every outer initial orbital period and output was generated every 100 outer orbital periods. A stopping condition was used to detect Roche-lobe overflow or a dynamical instability (Pelupessy et al. 2013).

In the event loop we first evolve all stars to the next time. In this step we apply the mass loss calculated for the tidal interaction from the previous step. We then perform the tidal evolution and recalculate the orbital elements and Cartesian coordinates of the three stars. Here we assumed that the argument of periastron and the mean anomaly are not affected by the tidal evolution. We finally integrate the gravitational dynamics of the triple system using the direct $N$-body code.

1 The AMUSE source code is public and can be downloaded via http: amusecode.org. The scripts used to perform the simulations described here are also available via the same website.
In Table. 1 we present an overview of initial conditions adopted in this experiment. We generally started with two inner stars of $M_{\mathrm{in}}^{\mathrm{A}}=110 \mathrm{M}_{\odot}$ and $m_{\mathrm{in}}^{\mathrm{A}}=30 \mathrm{M}_{\odot}$ in a $a_{\mathrm{in}}^{\mathrm{A}}=1 \mathrm{AU}$ orbit with an eccentricity of $e_{\mathrm{in}}^{\mathrm{A}}=0.1$. The $30 \mathrm{M}_{\odot}$ outer star orbits the inner binary in an $a_{\text {out }}^{\mathrm{A}}=25 \mathrm{AU}$ orbit with an eccentricity of $e_{\mathrm{out}}^{\mathrm{A}}=0.2$ at an inclination of $i^{\mathrm{A}}=90^{\circ}$. We also performed simulations with a $M_{\mathrm{in}}^{\mathrm{A}}=90 \mathrm{M}_{\odot}$ and a $M_{\mathrm{in}}^{\mathrm{A}}=150 \mathrm{M}_{\odot}$ inner primary star or with an eccentricity of the outer orbit of $e_{\text {out }}^{\mathrm{A}}=0.6$ and $a_{\text {out }}^{\mathrm{A}}=24 \mathrm{AU}$, with qualitatively gave the same results, although the moment of Roche-lobe overflow varies somewhat.

In Tab. 1 we notice that the time for reaching Rochelobe overflow for most of the calculated triples is considerably longer than the classical Lidov-Kozai period, which is caused in part by the coupled tidal and dynamical evolution, and in part by the requirement that Roche-lobe overflow should ensue. There may, however, still be a numerical effect, in particular in our assumption that tidal evolution is instanteneous at regular time intervals (of the outer orbital period) after which we progress this information to the rest of the system under the assumption that the argument of periastron and the orbital phase are not affected. If the outer orbit has a rather high eccentricity the collision tends to be established much earlier than when the outer orbit has a relatively small eccentricity. The choice of a high $\left(i^{\mathrm{A}} \sim 90^{\circ}\right)$ inclination is motivated by our follow-up study on the probable conditions before the common envelope (see $\S 4$ in which we discuss Markov-chain Monte-Carlo simulations to reconstruct the parameters of the outer orbit prior to the common envelope).

In Fig. 1 we present the orbital evolution of one of the calculations until the moment that the inner primary fills its Roche-lobe. In this simulation the merger was initiated by a Lidov-Kozai cycle that started about $0.255 \mathrm{Myr}$ after the birth the system and brought the eccentricity to a maximum of 0.863 . The subsequent tidal interaction lasted for about 180 yrs during which the semi-major axis was reduced to $a_{\text {in }} \simeq 0.563 \mathrm{AU}$ with an eccentricity $e_{\text {in }} \simeq 0.66$.

\subsection{Tidal energy dissipation and the possible origin of the Homonculus}

During the $\sim 180$ year epoch before the common envelope much energy is generated in the primary star by tidal dissipation. Fig. 2 depicts the tidal energy generated in this star as a function of time prior to the merger. The figure shows that during the last 50 years prior to the merger the tidal energy input into the star is of the order of $(4-6) \times 10^{39} \mathrm{erg} / \mathrm{s} \simeq 1-1.5 \times 10^{6} \mathrm{~L}_{\odot}$.

Already without this extra energy input the luminosity of this star is close to the Eddington luminosity. Owocki \& Shaviv (2012) have argued that if due to an extra energy source temporarily the luminosity exceeds the Eddington limit, very large continuum-radiation-driven mass loss will set in, leading to an eruptive phase, with an extremely high mass loss rate. This is because a continuum-driven wind can in principle lead to mass-loss rates up to the "photontiring limit", for which the entire luminosity is expended in lifting the atmosphere. We therefore propose here that the Homonculus, with a mass of about $20 \mathrm{M}_{\odot}$, was produced in an eruptive phase of a few decades that resulted from the huge tidal energy input in the star prior to the merger. 
Table 1. Initial conditions and results of the dynamical simulations with stellar evolution and tidal interaction (phase A). The secondary star of the inner binary $\left(m_{\mathrm{in}}^{\mathrm{A}}\right)$ as well as the mass of the tertiary star $\left(m_{\text {out }}\right)$ are fixed to $30 \mathrm{M}_{\odot}$. The inclination between the inner and the outer orbit was $i^{\mathrm{A}}=90^{\circ}$. The first 6 columns give the other initial conditions, primary mass $\left(M_{\mathrm{in}}^{\mathrm{A}}\right)$, fraction of tidal energy that drives the mass loss ( $f$ between 0 and 1), orbital parameters (semi-major axis $a_{\mathrm{in}}^{\mathrm{A}}$ and eccentricity $e_{\mathrm{in}}^{\mathrm{A}}$ ) of the inner binary, followed by the parameters of the outer binary $\left(a_{\text {out }}\right.$ and $\left.e_{\text {out }}\right)$. The subsequent columns give the results of the simulations, starting with the moment of highest eccentricity $\left(t_{\max , \mathrm{e}}\right)$, the associated eccentricity reached $\left(e_{\mathrm{in}, \max }\right)$ and the maximal mass loss rate due to tidal interaction $\left(\dot{M}_{\max }\right)$. Subsequent columns give the time between reaching the maximal eccentricity and the moment of Roche lobe overflow $\left(d t_{\mathrm{RLOF}}\right)$, the semi-major axis $\left(a_{\mathrm{RLOF}}\right)$ and eccentricity $\left(e_{\mathrm{RLOF}}\right)$ of the inner binary at RLOF, and the mass of the donor at this moment $\left(M_{\mathrm{RLOF}}\right)$. The outer orbit, not listed in the table, is only slightly affected by the mass loss of the primary star (see Portegies Zwart 2013, for a qualitative discussion on this).

\begin{tabular}{|c|c|c|c|c|c|c|c|c|c|c|c|c|c|}
\hline $\begin{array}{l}M_{\text {in }}^{\mathrm{A}} \\
\mathrm{M}_{\odot}\end{array}$ & $f_{\text {tidal }}$ & $\begin{array}{l}a_{\mathrm{in}}^{\mathrm{A}} \\
\mathrm{AU}\end{array}$ & $e_{\mathrm{in}}^{\mathrm{A}}$ & $\begin{array}{l}a_{\text {out }} \\
\mathrm{AU}\end{array}$ & $e_{\text {out }}-$ & $\begin{array}{l}t_{\max , \mathrm{e}} \\
\mathrm{Myr}\end{array}$ & $e_{\mathrm{in}, \max }$ & $\begin{array}{l}\dot{M}_{\max } \\
\mathrm{M}_{\odot} / \mathrm{yr}\end{array}$ & $\begin{array}{l}d t_{\text {RLOF }} \\
\mathrm{yr}\end{array}$ & $\begin{array}{l}a_{\mathrm{RLOF}} \\
\mathrm{R}_{\odot}\end{array}$ & $e_{\mathrm{RLOF}}$ & $\begin{array}{r}M_{\mathrm{RLOF}} \\
\mathrm{M}_{\odot}\end{array}$ & Figure \\
\hline 90 & 1 & 1.0 & 0.1 & 25.0 & 0.6 & 0.242 & 0.82 & 0.591 & 291 & 0.35 & 0.67 & 27.7 & \\
\hline 110 & 0.5 & 1.0 & 0.1 & 25.0 & 0.2 & 0.255 & 0.85 & 0.192 & 237 & 0.56 & 0.68 & 98.8 & see Figs. 1,2 and 3 \\
\hline 110 & 0.250 & 1.0 & 0.1 & 25.0 & 0.2 & 0.256 & 0.86 & 0.063 & 177 & 0.57 & 0.66 & 87.8 & \\
\hline 110 & 0.125 & 1.0 & 0.1 & 25.0 & 0.2 & 0.256 & 0.85 & 0.025 & 225 & 0.59 & 0.69 & 105.0 & \\
\hline 110 & 0.0 & 1.0 & 0.1 & 25.0 & 0.2 & 0.255 & 0.84 & 0.000 & 118 & 0.78 & 0.72 & 110.0 & \\
\hline 110 & 0.5 & 1.0 & 0.1 & 24.0 & 0.6 & 0.006 & 0.79 & 0.149 & 297 & 0.50 & 0.61 & 89.1 & \\
\hline 110 & 0.5 & 1.0 & 0.1 & 20.0 & 0.2 & 0.086 & 0.64 & 0.108 & 683 & 0.57 & 0.62 & 98.1 & \\
\hline 110 & 0.5 & 1.0 & 0.1 & 22.0 & 0.2 & 0.111 & 0.47 & 0.210 & 588 & 0.42 & 0.46 & 98.5 & \\
\hline 110 & 0.5 & 1.0 & 0.1 & 24.0 & 0.2 & 0.224 & 0.86 & 0.127 & 228 & 0.61 & 0.68 & 89.9 & \\
\hline 110 & 0.5 & 2.0 & 0.1 & 25.0 & 0.2 & 0.011 & 0.90 & 0.252 & 213 & 0.53 & 0.65 & 85.0 & \\
\hline 110 & 0.5 & 2.0 & 0.1 & 25.0 & 0.6 & 0.004 & 0.90 & 1.218 & 103 & 0.44 & 0.59 & 77.4 & \\
\hline
\end{tabular}

In this phase the star is very rapidly rotating and oblate. Owocki \& Shaviv (2012) have argued that in such a case the radiation-driven wind is much stronger in the polar than in the equatorial regions of the star, leading to a structure like the Homonculus. Therefore, the axis of the Homonculus is expected to be along the rotation axis of the primary star, which is perpendicular to the orbital plane of the inner binary.

\subsection{Evolution up to the merger}

The enhanced mass loss during the tidal event causes the primary star to shrink from $18.4 \mathrm{R}_{\odot}$ (before the strong tidal interaction), down to $14.5 \mathrm{R}_{\odot}$ with a rotational frequency of $\Omega \simeq 2.71 \times 10^{-5} \mathrm{~s}^{-1}$ when the highest orbital eccentricity is reached, which causes the star to swell to exceed $29.3 \mathrm{R}_{\odot}$ at the equator, at which time the two stars engage in a common envelope and coalesce.

The mass loss of the primary star prior to the merger, has caused the outer orbit to expand slightly from $a_{\text {out }}=$ $25 \mathrm{AU}$ to $a_{\text {out }} \simeq 28.1 \mathrm{AU}$ but has a smaller effect on eccentricity of the outer orbit $e_{\text {out }} \simeq 0.17$ (starting from $e_{\text {out }}=0.20$ ).

Each of our simulations of the evolution of the triple results in the Roche-lobe overflow of the primary star. The inner binary is unstable against mass transfer from the primary to the secondary star due the small mass ratio (in most cases $q_{\text {in }} \lesssim 1 / 3$ ), which causes rapid shrinking of the orbit. This, results in a common-envelope phase leading to coalescence. This coalescence between the inner two stars is quite a violent event, the consequences are far reaching for the entire triple. The energy released in such a merger process can measure about $8-12 \times 10^{49}$ erg (Soker \& Kashi 2013), which is sufficient to explain the great eruption (Rest et al. 2012). In addition, the mass lost in the merger process is likely to be asymmetric (Morris \& Podsiadlowski 2006), with has quite specific consequences for the surviving binary system, which after the merger is composed of the merger product and the tertiary star.

To further quantify the merger event we perform smoothed particles hydrodynamical simulations of the collision, which we describe in $\S 3$.

\section{ETA CARINAE DURING THE GREAT ERUPTION (PHASE B)}

We quantify the consequences of the coalescence by means of smoothed particles hydrodynamics simulations of the merger process, again using AMUSE. We are in particular interested in the amount of mass that is ejected during the merger process, the direction in which this mass is ejected, the resulting magnitude and the direction of the velocity kick that is induced upon the merger product, the morphology of the ejecta and the consequences for the orbital parameters of the outer star.

The hydrodynamical simulations are performed using a binary system in which the more massive primary star (with mass $M_{\text {in }}$ ) and the less massive secondary star (with mass $m_{\text {in }}$ ) orbit each other with semi-major axis $a_{\text {in }}$ and eccentricity $e_{\text {in }}$. For now we ignore the tertiary star, because it hardly plays a role in the merger process, and we will 


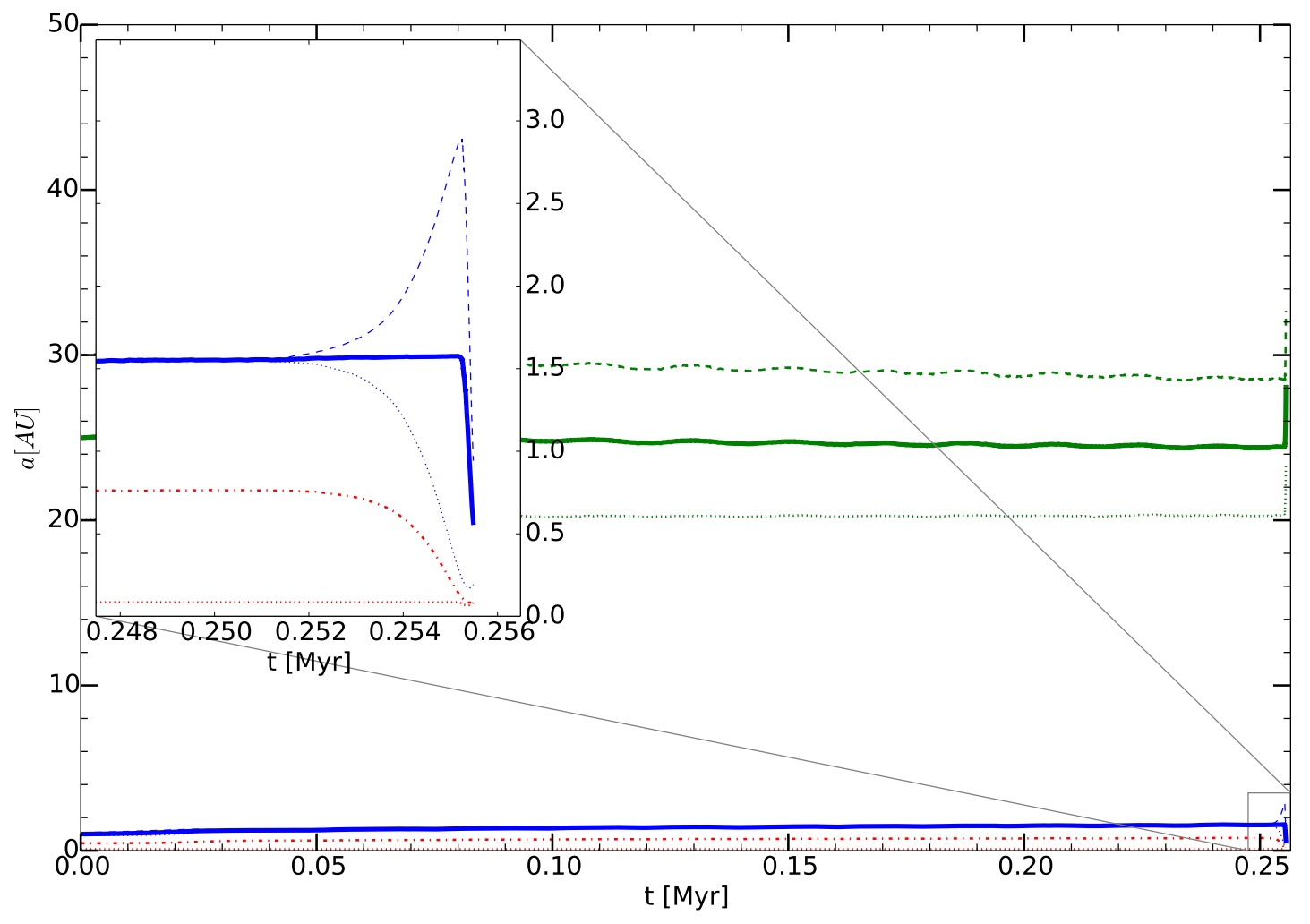

Figure 1. Evolution of the orbital separation of an example triple system, described in the text. The inner binary initially had a semi-major axis of $a_{\text {in }}^{\mathrm{A}}=1 \mathrm{AU}$ and eccentricity $e_{\mathrm{in}}^{\mathrm{A}}=0.1$ with primary and secondary masses of $M_{\mathrm{in}}^{\mathrm{A}}=110 \mathrm{M}_{\odot}$ and $m_{\text {in }}^{\mathrm{A}}=30 \mathrm{M}_{\odot}$. The outer orbit is inclined by $i^{\mathrm{A}}=90^{\circ}$ and has $a_{\text {out }}=25 \mathrm{AU}$ and $e_{\text {out }}=0.2$ with a tertiary mass of $m_{\text {out }}=30 \mathrm{M}_{\odot}$. The top three curves (green) give the evolution of the outer orbital apocenter, semi-major axis and pericenter (from top to bottom). The bottom three blue curves (better visible in the inset to the top left) give the same parameters for the inner orbit. The dashed-dotted curve (red, second from bottom) gives an estimate of the instantaneous radius of the Roche lobe of the inner primary star (Sepinsky et al. 2007), and the bottom curve (dotted red) gives the radius of this star. The primary overfills its Roche lobe after 255,490 yr.

explore the effect of the merger on the tertiary star further in $\S 4$.

After the initial conditions are selected, the primary and secondary stars are evolved using MESA. We first evolve the inner primary star up to the moment that the dynamical evolution determines the onset of Roche-lobe overflow (see $\S 2.1$ ). In our calculations in which the primary was born as a $110 \mathrm{M}_{\odot}$ star, it is reduced through the tidal evolution massloss to about $87.6 \mathrm{M}_{\odot}$. By the time of Roche-lobe overflow at $t \simeq 255 \mathrm{kyr}$ it's radius measures $29.3 \mathrm{R}_{\odot}$, which is considerably larger than its equilibrium radius of $18.4 \mathrm{R}_{\odot}$. The tidal interaction has further increased the star's rotational frequency to $\Omega \simeq 2.71 \times 10^{-5} \mathrm{~s}^{-1}$ (see $\S 2.1$ for details). The companion star is evolved to the same age and we adopted a rotational frequency of $\omega=2.6 \times 10^{-6} \mathrm{~s}^{-1}$.

After the dynamical evolution of the triple (see $\S 2.1$ ) and upon Roche-lobe overflow both stars in the inner binary are converted to a hydrodynamic particle representation in order to be able to continue the evolution with an smoothed particles hydrodynamics (SPH) code. The conversion from a 1-dimensional stellar density and composition profile in the Henyey code to the 3 dimensional particle representation as is used in an SPH code was done with the method available in AMUSE, which is described in de Vries et al. (2014). All $\mathrm{SPH}$ particles have the same mass. In this process the SPH particles are initially placed on a grid, which is subsequently relaxed by running an SPH code for 10 dynamical time scales of the primary star. We adopted the Gadget2 SPH code (Springel 2005) for this relaxation process.

The two hydrodynamcal stars are subsequently placed in the pre-determined binary orbit (see $\S 2.1 \$ 2.3$ and Tab. 2 ) and the evolution of the system is continued. For the latter we adopted the SPH code Fi (Pelupessy et al. 2004). Changing codes in AMUSE is self-consistent and easy: it requires changing a single line in the Python-AMUSE script. Running the relaxation of the stars with one SPH code and the subsequent gravitational/hydrodynamical evolution of the system with another is therefore motivated by performance and applicability of the particular code for that specific task. The Fi SPH code is, in this case, better suited for simulating the merger process, whereas GadGet2 was already adopted and extensively tested in de Vries et al. (2014) for converting Henyey stellar evolution models to SPH realizations.

We adopted the parameters for the inner orbit just be- 


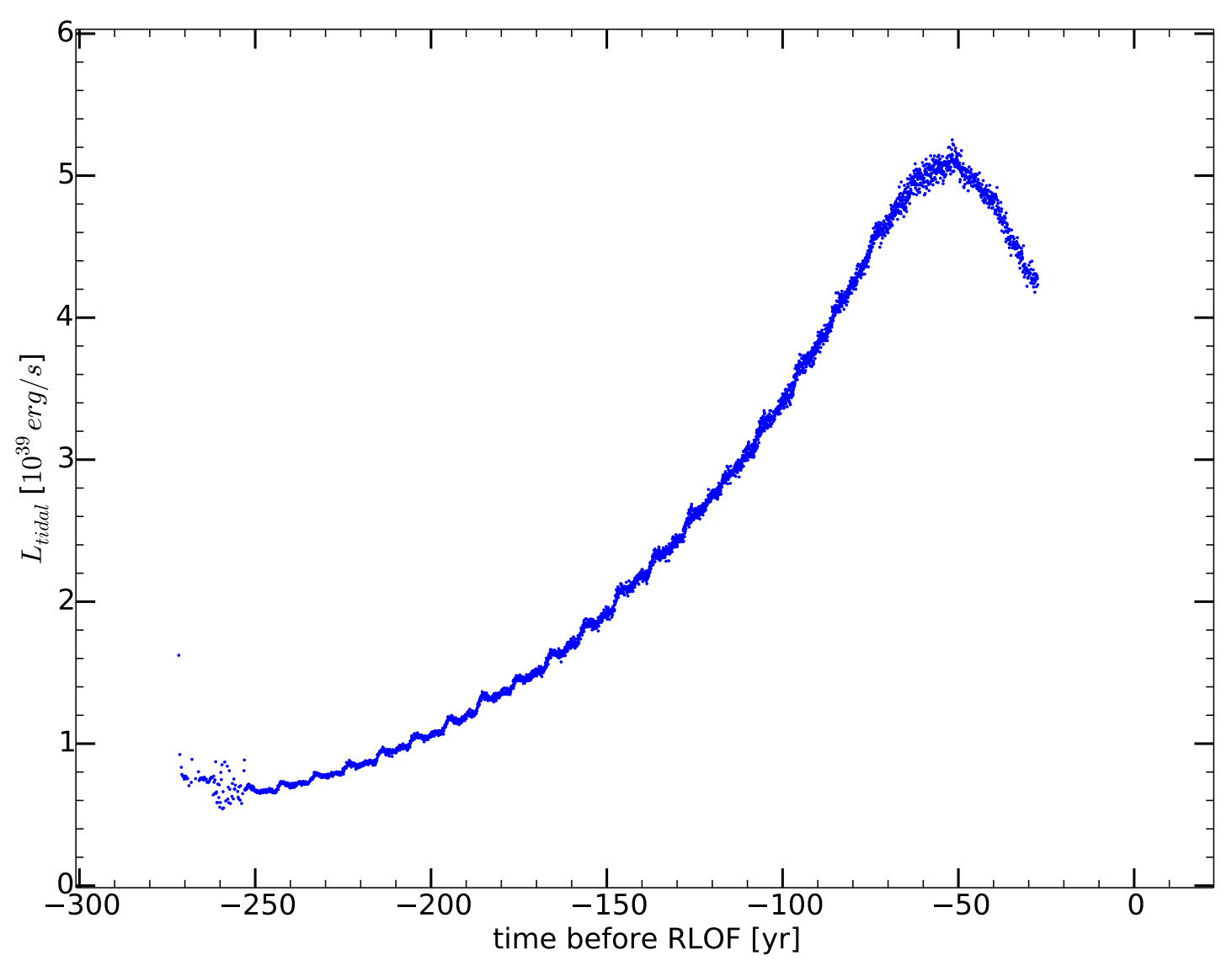

Figure 2. Tidal energy dissipated in the donor star (of the inner binary system) prior to Roche-lobe overflow. Here Roche-lobe overflow occurs at $t=0 \mathrm{yr}$ (which corresponds to $255,490 \mathrm{yr}$ after the birth of the system at the zero-age main-sequence). The results are from the same simulation as presented for the orbital evolution in Fig. 1 . As explained in $\S 2.2$ we expect that due to the large tidal energy input the luminosity of the star to exceed the Eddington luminosity, leading to a huge continuum-drive wind mass loss of order $20 \mathrm{M}_{\odot}$ in a few decades prior to the merger, which we suggest to have produced the Homonculus nebula.

fore the merger from the dynamical simulations described in $\S 2.1$ (and Tab. 2). From the moment of first contact at pericenter, the two stars orbit each other up to a dozen times before the merger is complete. In fig. 3 we present the evolution of the semi-major axis of one of these models.

In some of our simulations the binary did not result in a merger within 100 days, after which we stopped them. The latter cases could eventually result in a merger, but the parameters would be different due to the continuing KozaiLidow cycles and stellar evolution, which we ignored in the $\mathrm{SPH}$ simulations, because we omitted the tertiary star in the hydrodynamical simulations.

The results of the SPH simulations are analyzed using HOP (Eisenstein \& Hut 1998) with the saddle-point density cut-off set to the mean density and the outer density threshold to $5 \%$ of the mean density. We continue running the SPH simulation until only one single blob of particles remains. After the first detection of a single blob, we continue to run the hydrodynamics for an orbital period of the outer star to assure that the single object really remains integer. This single blob is considered to be the merger product, and the residual material is identified as the ejecta in the merger process.

We performed several convergence tests, one is presented in fig. 4. Here we show the mass of the merger product as a function of the number of SPH particles. The mass of the merger product seems to converge at $\gtrsim 10^{4} \mathrm{SPH}$ particles for the primary star; lower resolution simulations systematically underestimate the amount of mass ejected in the merger process. We performed similar analyses for the time or the merger, the velocity of the ejecta and the merger product, and for the direction and morphology of the ejected material. Each study shows convergence for $\gtrsim 10^{4} \mathrm{SPH}$ particles.

\subsection{The stellar collision merger product}

We realize that the number of simulations we performed is rather limited, and can hardly be considered a complete coverage of parameter space, but we think that our simulations are representative for the physical process we try to describe. 
Table 2. Initial conditions and results for the smoothed particles hydrodynamics simulations. The first column gives the number of SPH particles for the primary star. The SPH particles in the simulations for the primary and secondary star have the same mass. and the total number of SPH particles is The following columns give the age of the system at the moment of Roche-lobe overflow, and the masses of the primary $\left(M_{\mathrm{in}}^{\mathrm{B}}\right)$ and secondary $\left(m_{\mathrm{in}}^{\mathrm{B}}\right)$ stars at that moment. In the fifth and sixth columns we give the initial orbital separation $a_{\mathrm{in}}^{\mathrm{B}}$ and eccentricity $e_{\mathrm{in}}^{\mathrm{B}}$ of the binary at the moment of Roche-lobe overflow. The last three columns give the duration of the merger process, the final total mass in the merger product $\left(M^{\mathrm{C}}\right)$ and its velocity $v_{\text {kick }}$ as a result of the asymmetric mass ejection during the merger process. In the SPH simulations we ignore the effect of the tertiary star, because the effect of Lidov-Kozai cycles on the inner orbit is negligible on the short time scale of the merger.

\begin{tabular}{rlllllllll}
\hline $\begin{array}{r}N_{\mathrm{SPH}} \\
\times 1000\end{array}$ & $\begin{array}{l}t_{\mathrm{RLOF}} \\
\mathrm{Myr}\end{array}$ & $\begin{array}{c}M_{\mathrm{in}}^{\mathrm{B}} \mathrm{M}_{\odot} \\
m_{\text {in }}^{\mathrm{B}}\end{array}$ & $\begin{array}{l}a_{\text {in }}^{\mathrm{B}} \\
\mathrm{R} \odot\end{array}$ & $e_{\text {in }}^{\mathrm{B}}$ & $\begin{array}{l}t_{\mathrm{mrg}} \\
\text { day }\end{array}$ & $\begin{array}{l}M^{\mathrm{C}} \\
\mathrm{M}_{\odot}\end{array}$ & $\begin{array}{l}v_{\text {kick }} \\
\mathrm{km} / \mathrm{s}\end{array}$ & Figure \\
\hline 10 & 0.96 & 70 & 30 & 77 & 0.87 & 54.3 & 77.3 & 193 & \\
50 & 0.96 & 70 & 30 & 77 & 0.87 & 17.3 & 68.8 & 81.5 & see Fig. 3 \\
100 & 0.49 & 80 & 20 & 77 & 0.87 & 26.4 & 83.1 & 70.6 & \\
10 & 0.49 & 80 & 20 & 77 & 0.87 & 21.2 & 83.8 & 58.6 & \\
50 & 0.49 & 80 & 20 & 77 & 0.87 & 15.5 & 83.2 & 61.7 & \\
50 & 0.13 & 90 & 10 & 77 & 0.87 & 27.4 & 90.1 & 211 & \\
2 & 0.49 & 80 & 20 & 77 & 0.87 & 7.5 & 86.3 & 34.8 & \\
4 & 0.49 & 80 & 20 & 77 & 0.87 & 11.1 & 84.7 & 12.6 & \\
8 & 0.49 & 80 & 20 & 77 & 0.87 & 13.0 & 83.7 & 30.3 & \\
16 & 0.49 & 80 & 20 & 77 & 0.87 & 22.3 & 83.6 & 52.7 & \\
20 & 0.49 & 80 & 20 & 77 & 0.87 & 7.1 & 78.0 & 56.5 & \\
32 & 0.49 & 80 & 20 & 77 & 0.87 & 7.9 & 83.6 & 56.9 & \\
32 & 0.49 & 80 & 20 & 77 & 0.87 & 7.9 & 83.4 & 56.9 & \\
64 & 0.49 & 80 & 20 & 77 & 0.87 & 8.5 & 77.5 & 47.7 & \\
128 & 0.49 & 80 & 20 & 77 & 0.87 & 8.9 & 77.8 & 54.5 & see Figs 5 \& 6 \\
256 & 0.49 & 80 & 20 & 77 & 0.87 & 5.8 & 78.1 & 60.9 & \\
16 & 0.47 & 124 & 30 & 77 & 0.87 & 2.8 & 138 & 25.1 & \\
32 & 0.47 & 124 & 30 & 77 & 0.87 & 3.2 & 137 & 18.6 & \\
16 & 1.82 & 88 & 30 & 121 & 0.92 & 9.5 & 100 & 12.3 & \\
32 & 1.82 & 88 & 30 & 121 & 0.92 & 7.8 & 101 & 14.5 & \\
\hline
\end{tabular}

We realize that we should not interpret the result of the SPH simulations statistically, but by a lack of understanding the initial parameter space we discuss them as a canonical ensemble. On average the merger takes $14 \pm 12$ days during which $18.3 \pm 4.3 \mathrm{M}_{\odot}$ is lost from the two inner stars. This mass is ejected by the violent in-spiral of the original inner binary system in $\mathrm{a} \sim 70^{\circ}$ shell centered around the direction of the apocenter of the inner orbit. In Fig. 5 we present the spatial morphology of the out flowing gas 8.8 days after the merger of our hydrodynamical simulation in which an $80 \mathrm{M}_{\odot}$ star merges with a $20 \mathrm{M}_{\odot}$ star from an $77 \mathrm{R}_{\odot}$ orbit with an eccentricity of 0.87 . The velocity of the ejected material is about $400-500 \mathrm{~km} \mathrm{~s}^{-1}$.

Earlier calculations arrive at similar conclusions regarding the velocity and direction of the ejecta (Thackeray 1961; Walborn et al. 1978). Due to conservation of momentum, the merger product receives a kick of $v_{\text {kick }}=76.4 \pm 53.9 \mathrm{~km} / \mathrm{s}$ in the plane and roughly in the direction of pericenter in the inner orbit.

Upon its subsequent pericenter passage, some 5.5 years later, the outer star plunges through the bloated merger product, giving rise to the 1843 outburst.

We have illustrated the chain of events that may have led to the great eruption of Eta Carinae. We will now demonstrate how the model may satisfactorily explain its observed orbital parameters by means of Markov-chain Monte Carlo simulations of the evolution of the triple through the merger process.

\section{RECONSTRUCTING THE TRIPLE TOPOLOGY DURING THE GIANT ERUPTION}

The parameter space for triples that engage in an evolution similar to that described in the previous section is rather large and simulating the secular evolution of the triple system and the subsequent merger are too expensive in terms of computer time to fully map parameter space. Ideally, we would like to perform a exhaustive search of parameters using our model in order to constrain the initial conditions and the events that led to the currently observed system, but that is not realistic. Instead, we construct a model of the consequences of the merger by making a few simplifying assumptions, and adopt a Markov-chain Monte Carlo approach to map parameter space and constrain the premerger parameters of the triple system.

This approach is separate and independent of the earlier calculations (see $\S 2$ and $\S 3$ ) and there is no guarantee that the results of the Monte Carlo simulations provide reasonable conditions prior to the merger. The fact that the model provides reasonable conditions bolsters our confidence in the proposed scenario for the giant eruption of Eta Carinae.

One of the assumptions we will make is that the time scale for the merger is short compared to the orbital period of the outer star in the triple system $(\sim 12 \mathrm{yr})$. The SPH simulations of the merger process could be viewed as a guideline for these time scales, which turn out to be $\lesssim 25$ days (see $\S 3$, Fig. 3 and Tab. 2). The merger itself is therefore perceived by the outer star as an impulsive event, in which the 


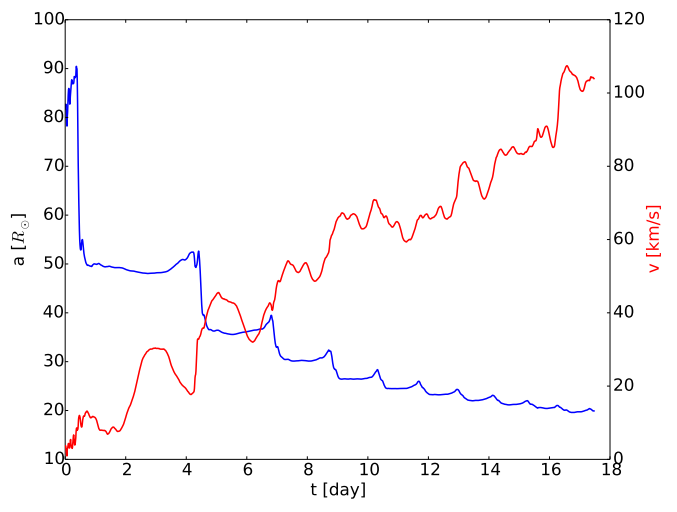

Figure 3. Orbital evolution of the inner binary until it merges (blue, axis to the left), and the velocity of the bound mass (red, axis to the right). These curves are the result of an SPH simulation using two stars of $M_{\mathrm{in}}^{\mathrm{B}}=70 \mathrm{M}_{\odot}$, and $m_{\mathrm{in}}^{\mathrm{B}}=30 \mathrm{M}_{\odot}$ at an age of $1.0 \mathrm{Myr}$ in a $a_{\mathrm{in}}^{\mathrm{B}}=77 \mathrm{R}_{\odot}$ and with eccentricity $e_{\mathrm{in}}^{\mathrm{B}}=0.87$. The $m_{\text {out }}=30 \mathrm{M}_{\odot}$ tertiary star has highly eccentric $e_{\text {out }}=0.9$ orbit of $a_{\text {out }}=100 \mathrm{AU}$ around the inner binary. The relative inclination and argument of pericenter are both $i^{\mathrm{B}}=90^{\circ}$. The Lidov-Kozai cycles induced by this outer star cause the eccentricity of the inner binary to grow to $e_{\text {in }}^{\mathrm{B}}=0.87$ (see phase A), upon which the two inner stars coalesce. The merger induces a kick of about $v_{\text {kick }}=81.5 \mathrm{~km} / \mathrm{s}$ onto the merger product, which resulted from the asymmetric ejection of the common-envelope of the inner binary. This kick was directed towards pericenter of the original inner binary. (Note, that this system is different than used in Fig. 2).

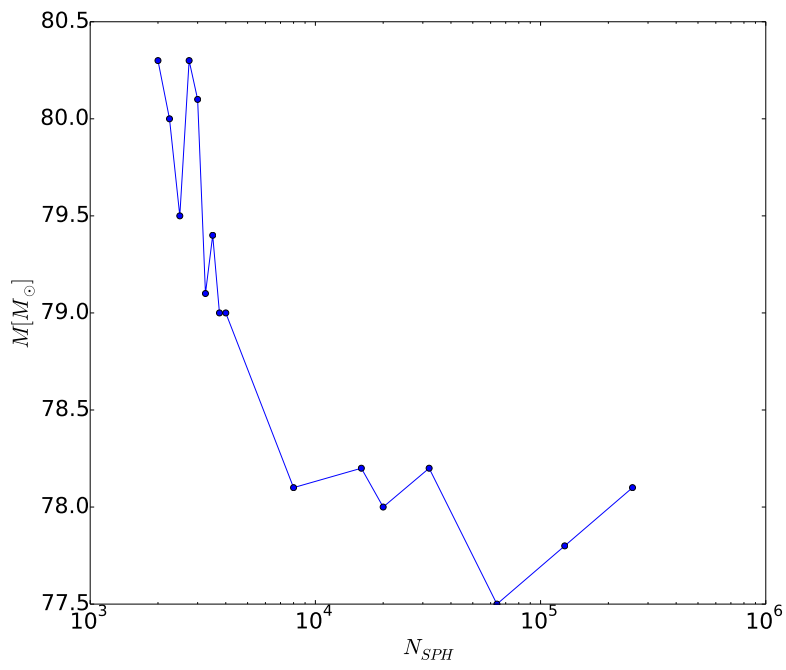

Figure 4. Mass of the merger product as a function of the number of SPH particles for the primary star. Each bullet point is one simulation using the same initial conditions but with varying number of SPH particles. The initial conditions at the moment of RLOF were $M_{\text {in }}^{\mathrm{B}}=90 \mathrm{M}_{\odot}, m_{\text {in }}^{\mathrm{B}}=30 \mathrm{M}_{\odot}, R_{\text {in }}=15 \mathrm{R}_{\odot}, a_{\text {in }}^{\mathrm{B}}=55 \mathrm{R}_{\odot}$ and $e_{\mathrm{in}}^{\mathrm{B}}=0.845$. The mass of the merger product converges to about $M^{\mathrm{C}}=78 \mathrm{M}_{\odot}$ which indicates at about $12 \mathrm{M}_{\odot}$ was ejected in the merger process.

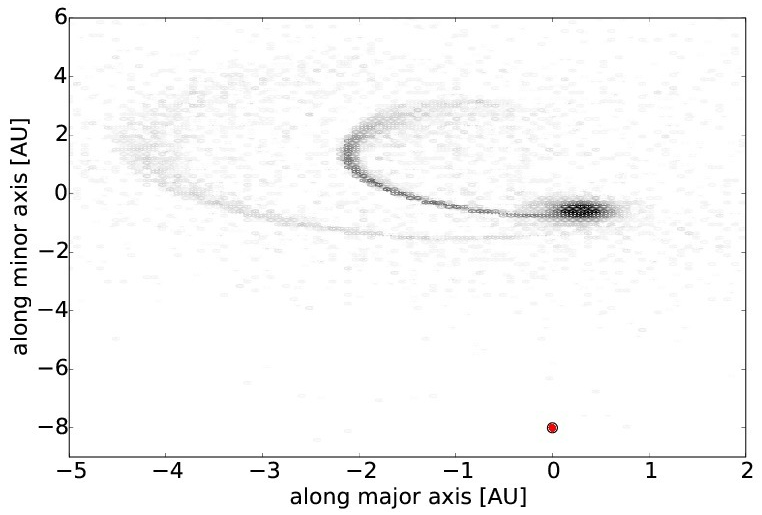

Figure 5. Projected density distribution (in gray) of the gas ejected in the merger process at the moment that the two stars in the inner binary have merged to one. The origin is in the original center of mass of the inner binary system. The two massive loops of ejected gas (to the left of the origin) are quite characteristic for the merger process. In Fig. 6 we present a more appealing picture of the same merger. At this moment the tertiary star will be near pericenter at the indicated position (red bullet) moving away from the reader. The hydrodynamical simulations are taken 8.8 days after the merger between a $M_{\text {in }}^{\mathrm{B}}=80 \mathrm{M}_{\odot}$ star and a $m_{\mathrm{in}}^{\mathrm{B}}=20 \mathrm{M}_{\odot}$ star in an $a_{\mathrm{in}}^{\mathrm{B}}=77 \mathrm{R}_{\odot}$ orbit with an eccentricity of $e_{\mathrm{in}}^{\mathrm{B}}=0.87$ (see Tab. 2).

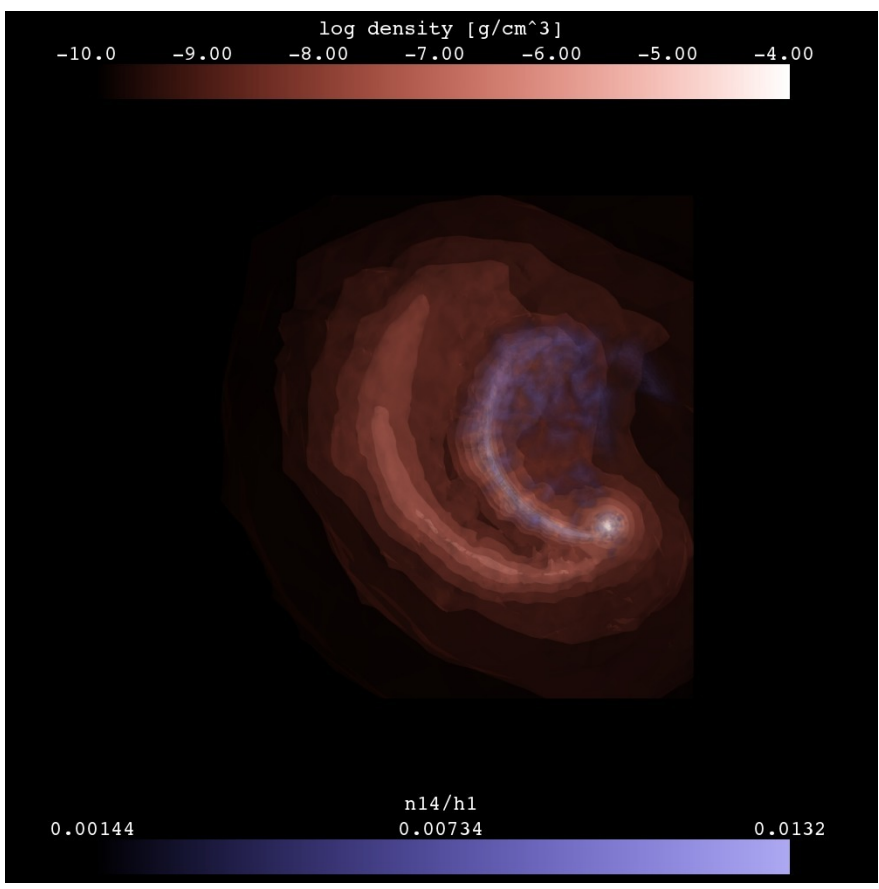

Figure 6. Same as Fig. 5 but then visually more attractive and in a rectangular frame of about $12 \mathrm{AU}$ at each side. The projected density is given in red, and the Nitrogen/hydrogen ratio is presented blue. 
inner binary merger loses mass and receives a velocity kick due to the asymmetry with which the mass is ejected. For the duration of the merger we further assume that the inner object (the merging binary star) is perceived by the outer star as a point mass.

The kinematics of the gas that is ejected in the merger process is less well constrained. The initial outer orbital period exceeds the initial inner orbital period by at least two orders of magnitude and it takes some time before the mass shell has passed the outer orbit. As we will discuss later, the merger event is likely to take place when the outer star is near its pericenter, which ensures that the time needed for the ejected mass shell to escape the (by then) binary system is small (30-70 days) compared to the orbital period of the outer star ( $\gtrsim 10$ years).

The time scale for the outer star to re-approach pericenter is about an orbital period of the post-merger binary system. This gives rise to a second outburst, some time after the 1838 merger event, which lasted several tens of days. This second outburst is then associated with the companion star plunging through the bloated merger product.

\subsection{Reconstructing the 1983 event}

We solve Kepler's equations for a two body system while taking the mass loss and the impulsive kick to the merger into account. Similar calculations have been performed in relation to supernovae in binary (Hills 1983; Tauris \& Takens 1998) and triple systems (Pijloo et al. 2012).

We adopt the Metropolis-Hastings algorithm (Hastings 1970) to reconstruct the initial pre-merger parameters of the triple system by using the currently observed orbital parameters for Eta Carinae as objective (see Tab. 3). The fixed parameters are the masses of the two stars in the binary after the merger $\left(M_{\mathrm{in}}^{\mathrm{B}}+m_{\mathrm{in}}^{\mathrm{B}} \equiv M^{\mathrm{C}}=90 \mathrm{M}_{\odot}\right.$ for the merger product and $m_{\text {out }}=30 \mathrm{M}_{\odot}$ for its companion).

The free parameters in the Markov chain are the total mass of the inner two stars before the merger, magnitude and direction of the velocity imparted to the merger product and the orbital parameters of the outer orbit $\left(a_{\text {out }}\right.$, $e_{\text {out }}$, orbital phase and relative inclination $i$ ) at the moment of merging. We search parameter space using the EMCEE (Foreman-Mackey et al. 2013) implementation for a Markovchain Monte Carlo simulations. The solution is degenerate, and therefore we run the Monte Carlo code several times in order to construct an ensemble of possible solutions. Each Monte Carlo realization is performed with 10 workers, a burn-in of 10 generations and with 200 subsequent iterations, which is sufficient for the solution to converge to within $1 \%$ of the observed parameters for Eta Carinae. In Fig. 7 we present the result of the Monte Carlo simulations, in particular the semi-major axis and eccentricity of the outer orbit prior to the merger event. This is supposedly the moment after the dynamical simulations described in $\S 2.1$ (phase A) but before the onset of the common-envelope evolution discussed in $\S 3$ (phase B).

The currently observed orbital parameters for the pregreat eruption binary system are best reproduced when the inner binary was orbited by another star in a relatively wide $a_{\text {out }}=20-100 \mathrm{AU}$ orbit with an eccentricity of $e_{\text {out }} \lesssim 0.7$. A total of $12-23 \mathrm{M}_{\odot}$ was lost, preferentially in the direction towards apocenter along the semi-major axis of the inner binary, inducing a velocity kick to the merger product of $v_{\text {kick }}=53 \pm 8 \mathrm{~km} / \mathrm{s}$ (with a minimum of $40 \mathrm{~km} / \mathrm{s}$ ) in the orbital plane of the inner binary and pointing to its pericenter $\left(\phi=180^{\circ} \pm 9^{\circ}\right)$ and almost perpendicular to the plane of the outer binary $\left(\theta=91^{\circ} \pm 21^{\circ}\right)$. This latter angle is interpreted as the relative inclination between the inner and the outer orbit. (Note that this finding motivated us in $\S 2.1$ to adopt inclinations $i^{\mathrm{A}}=90^{\circ}$.) The required mass loss and velocity kick are consistent with our earlier results from the hydrodynamical calculations presented in Table $2\left(18.3 \pm 4.3 \mathrm{M}_{\odot}\right.$ and $v_{\text {kick }}=61 \pm 51 \mathrm{~km} / \mathrm{s}$, see $\S 3$ ). The orbit of the outer star has, at the moment of the merger, a mean anomaly of $\mathcal{M}=0.061 \pm 0.035$, which is just barely past pericenter.

Such a highly inclined outer orbit induces Lidov-Kozai (Kozai 1962) cycles onto the inner orbit with a period of $\gtrsim 1 \mathrm{kyr}$, and drives the eccentricity of the inner orbit to exceed $\sim 0.97$. For tight inner orbits, $a_{\text {in }}^{\mathrm{A}}=0.30-0.6 \mathrm{AU}$, these cycles can last for as long as 1 Myr. Long periods (and tight inner orbits) are consistent with the observed age of Eta Carinae, but are possibly less suitable for inducing a collision via Lidov-Kozai cycles, because the variation in the orbital eccentricity (and therefore in the pericenter distance) per inner revolution is relatively small. However, as we discussed in $\S 2.1$, the response of the donor to the tidal evolution may be quite important for the merger process. In our simulations in $\S 2$ we discussed the growth of the tidally heated inner primary star, which is likely to initiate the merger.

A collision is more probable when the inner orbit is larger $1-2 \mathrm{AU}$, because this leads to large changes in the pericenter distance within one inner orbital revolution (see Fig. 7). Considering the large uncertainty in the age determination of Eta Carinae, we see sufficient leeway to argue that the system is considerably younger $(\lesssim 0.4 \mathrm{Myr})$ than $1 \mathrm{Myr}$. We therefore have a slight preference for relatively wide inner orbits, which motivated us to perform most of the simulations in $\S 2.1$ with $a_{\text {in }} \simeq 1 \mathrm{AU}$.

\section{DISCUSSION}

We have explored the possibility that the great eruption of Eta Carinae was initiated by a tidal interaction followed by the common envelope merger of the two stars of the inner binary in a hierarchical triple system. We support theoretical arguments with numerical simulations of various stages of the process. We find consistency with a number of observables, including various phases of fast and slow mass loss, the brightening of the object prior to the great eruption, and the events are consistent with the current orbital parameters of the surviving binary system. Encouraged by the successes, we will discuss some of these advantages of our model qualitatively and also raise some concerns.

\subsection{The brightening of Eta Carinae prior to the great eruption}

Our merger model can, at least qualitatively, account for the gradual increase in brightness of Eta Carina in the decades preceding the large outbursts of 1838-1843. During the first half of the nineteenth century (and possibly even in the two centuries before that) the visual brightness of Eta Carina 


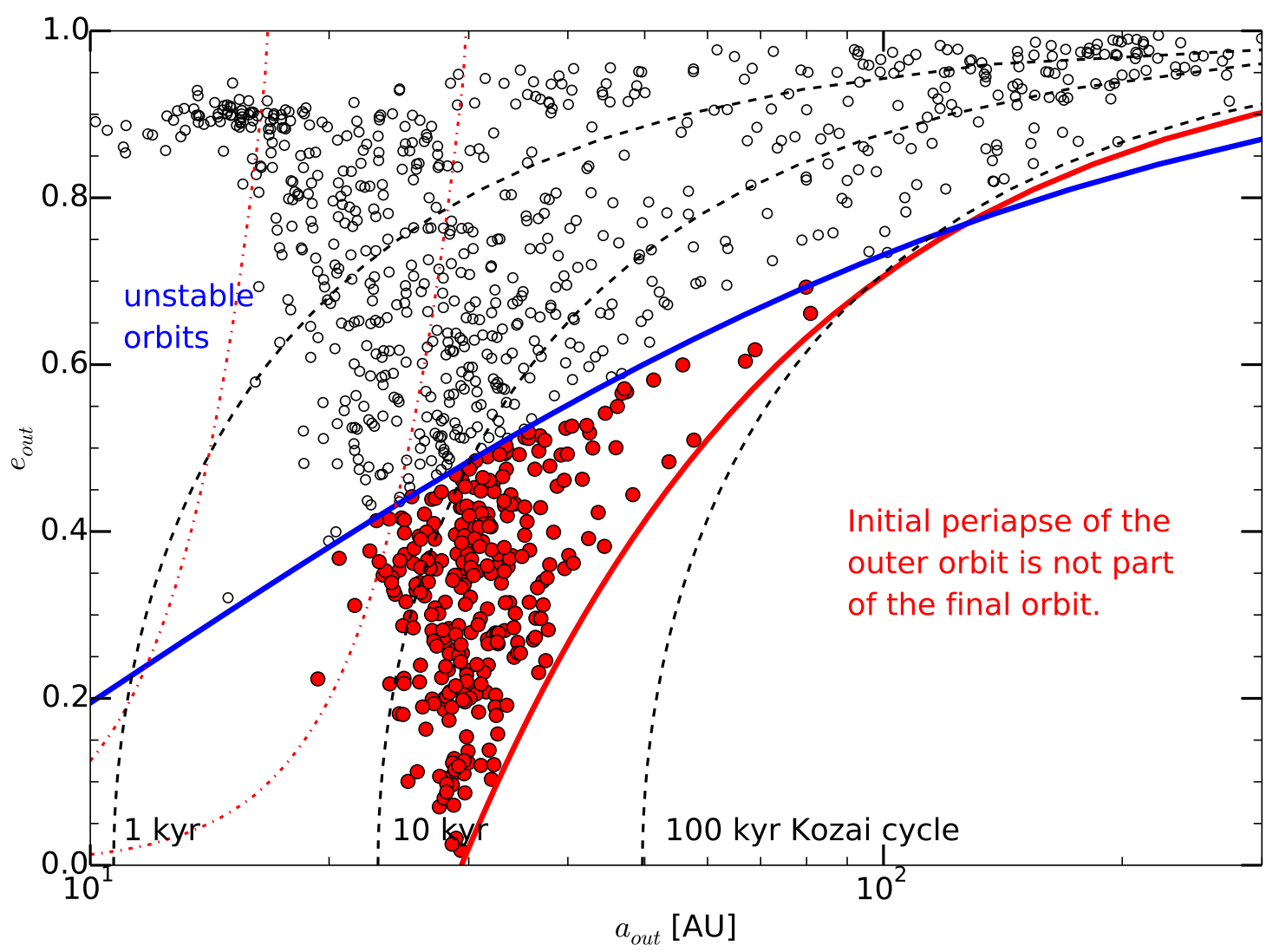

Figure 7. Range of solutions for the progenitor outer binary that can result in consistent currently observed orbital parameters of Eta Carinae. We adopted the inner stars of $M_{\mathrm{in}}^{\mathrm{B}}=90 \mathrm{M}_{\odot}$ and $m_{\mathrm{in}}^{\mathrm{B}}=30 \mathrm{M}_{\odot}$ in a circular inner orbit of $a_{\mathrm{in}}^{\mathrm{B}}=1 \mathrm{AU}$, the outer star was $m_{\text {out }}=30 \mathrm{M}_{\odot}$. Parameter space for viable solutions to the Lidov-Kozai induced merger. The dashed curves give an estimate of the time scale for Kozai cycles, from left to right $1 \mathrm{kyr}, 10 \mathrm{kyr}$ and $0.1 \mathrm{Myr}$, respectively. The red dash-dotted curves indicate parameters for which the pericenter distance was reduced due to the Lidov-Kozai effect by more than $0.1 \%$ and $0.01 \%$ (from left to right) of the radius of the secondary between two subsequent pericenter passages (Ford et al. 2000). The red solid curve gives the minimum distance in the orbit of the outer star such that it is part of the currently observed orbit of Eta Carinae: $p=R_{\text {sum }} / a_{\text {EtaCar }}\left(1+e_{\text {EtaCar }}\right)$. Here $R_{\text {sum }}$ is the sum of the radii of the inner two stars. This gives a minimal orbital separation for which an impulsive event can lead to a viable observed orbit. Initial outer orbits below this curve have no part of the orbit that is consistent with the currently observed orbit of Eta Carinae, and therefore do not lead to possible solutions. Systems above the blue solid curve are dynamically unstable at the moment of Roche-lobe overflow (Mardling 2008), and therefore do not provide a possible solution. Only those systems (red bullets) between the solid blue and the solid red curve can lead, through a merger event, to a system with parameters consistent with the currently observed orbit of Eta Carinae (see Tab. 3). The circles give the result of the Monte-Carlo simulations aiming to reproduce the current orbital parameters ( $a_{\mathrm{EtaCar}}$ and $\left.e_{\mathrm{EtaCar}}\right)$ for Eta Carinae. The open symbols give dynamically unstable solutions, whereas the bullets give stable solutions.

had increased to magnitudes 4 or brighter, from a quiescent value of $\sim 8$ magnitudes, which it assumed again after year 1900 (although after 1940 it brightened again to about visual magnitude 5 , which has been ascribed to dust destruction and expansion of a dust envelope Humphreys \& Martin (2012)). This large visual luminosity increase cannot be solely explained by a bolometric correction effect of a star with an expanding photospheric radius at constant bolometric luminosity: this may explain at most perhaps an increase of 2.5 to 3 visual magnitudes. Therefore it appears that the bolometric luminosity of Eta Carina already increased considerably in the centuries preceding the giant outburst, which means that somehow extra energy must have been generated in its interior during this period. Hydrogen fusion in stellar interiors tends to be highly stable and self-limiting (hydrogen-fusing stars have an in-built feedback "safetyvalve": a slight overproduction of nuclear energy leads to increase in core temperature, leading to increased core gas pressure, which will make the core expand and adiabatically cool, quenching the increased rate of fusion Eggleton 2006). It therefore seems highly unlikely that this increase would be due to increased nuclear fusion in the interior as has, for example, been suggested by Owocki \& Shaviv (2012). (It should be noticed, however, that in stellar interiors where radiation pressure plays a dominant role the feedback loop of fusion has not yet been well studied).

We argue here that the increased bolometric luminosity preceding the large outburst is due to tidal friction produced 
by the in-spiralling companion star. In $\S 2$ we estimate the energy that can be generated by this friction, which shows that it may, in principle, lead to an energy release comparable to the luminosity increase preceding the giant eruption. We argued there that since the unperturbed star is already very close to its Eddington luminosity, a large increase of its luminosity due to tidal friction is in itself sufficient to cause a phase of eruptive mass-loss that could have produced the Homonculus.

\subsection{The surface abundances of Eta Carinae}

Eta Carinae has an enhanced atmospheric N and He abundances and reduced $\mathrm{C}$ and $\mathrm{O}$ abundances (Humphreys \& Martin 2012). The surface abundance of a star can be calculated using our adopted Henyey stellar evolution code (see $\S 2.1$ ). We perform this calculation by measuring the abundances in the outer-most shell in the Henyey code for the $110 \mathrm{M}_{\odot}$ zero-age star in the triple calculation of $\S 2.1$ at the moment of RLOF. Upon RLOF the mass of the star was reduced due to the tidally induced stellar wind to about $87.6 \mathrm{M}_{\odot}$. We compare the surface abundance with the resulting surface composition of the merger product of our hydrodynamical simulations in $\S 3$. For the latter we measure the abundances of the outer most $1 \%$ of the SPH particles in the simulations with $N_{\mathrm{SPH}}=128,000$. The former measurement has no formal error, whereas the result of the SPH calculations has a Poissonian error.

The merger process leads to a slight enhancement in the helium abundance, from a relative fraction of 0.28 in the Henyey code to $0.295 \pm 0.018$ after the merger simulation; a relative increase of $5.4 \%$. Nitrogen however, is enhanced through the merger process by a factor 3.7 (from 0.00101 to $0.0047 \pm 0.0034)$. Our measured nitrogen enhancement however, is still about a factor of 2 smaller than the observed enhancement (Melendez et al. 2005). Oxygen in our simulations is depleted by $19 \%$ (from 0.0094 to $0.0076 \pm 0.0018$ ), which is smaller than the observed depletion (Melendez et al. 2005).

We have not further studied the effect of the surface abundances as a function of the parameters. The trends of enhanced nitrogen and depleted oxygen is promising, and we think that the relatively small deviations from the observations can be matched by the simulation with a more exhaustive exploration of the initial parameter space. We performed only a very limited parameter study, and a more exhaustive study may be used to reduce the uncertainty in our adopted initial conditions.

\subsection{The morphology of the outflow produced by the merger}

Apart from the "Homunculus" nebula the entire region around Eta Carinae has a rather unstructured appearance (see also Smith \& Townsend 2007). Although our model of a binary merger while orbited by a third companion tends to drive an asymmetric outflow, at least as a result of the binary merger itself, there is also a considerable component in which the outflow is symmetric. In particular in the episode of highly enhanced wind loss prior to the merger, as was discussed in sections 2.2 and 5.1 , we expect to have produced a symmetric bi-modal structure. We therefore think that the observed symmetries in the Homunculus do not pose a serious limitation for our model (any post-merger disturbance of the primary, e.g. by periastron passage of the companion, will again lead to a Homonculus-like structure, such as the Little Homonculus).

It should be noticed here that the merger product will rotate with break-up velocity. Due to the Von Zeipel theorem (von Zeipel 1924) the polar regions of this star will therefore be very much hotter than its equatorial regions, which is expected to lead to very much stronger radiatively driven stellar wind mass loss from the polar regions than from the equatorial regions. We suggest that this has produced the Homunculus structure of Eta Carinae (see also Maeder 1999; Aerts et al. 2004).

From interferometric measurements Weigelt \& Kraus (2012) noticed that, apart from the bipolar-shaped Homunculus there are slow-moving "knots" - condensations within 0.3 " from the central star that are moving at $v \simeq 50$ $\mathrm{km} / \mathrm{s}$, and apparently have been ejected in or soon after the great eruption, and seem ejected more or less perpendicular to the axis of the bipolar nebula: in the equatorial plane of the Homunculus.

This is confirmed by Weis' (2012) study of the HST and CHANDRA images of the surroundings of the star. Weis notices that, while the Homunculus with its two lobes (and equatorial "skirt" of $\lesssim 1 \mathrm{M}_{\odot}$ ) has a near-perfect bipolar symmetry, the outer ejecta that presumably date from the pre-1838 tidally induced dense stellar wind, appears much more asymmetric, and is composed of many irregularly-shaped structures in a roughly elliptically-shaped region around the Homunculus, the axis of the ellipse being perpendicular to the axis of the latter: the largest are the Scondensation and the S-ridge (a large highly asymmetricallyshaped structures on one side of the Homunculus). This condensation as well as the NN-knots on the opposite side of the Homunculus were apparently ejected perpendicular to the axis of the Homunculus (in its equatorial plane). The fact that these ejecta are nitrogen enriched indicate that CNO-processed material was brought to the surface and was violently ejected in the great eruption. Such nitrogen enrichment is a natural consequence of the proposed merger scenario (see also $\S 5.2$ ), in which we presented the result of one of our SPH simulation of the merger, and where we indicate the presence of nitrogen enhanced material. Weis (2012) notices that these outer ejecta are more or less a conglomerate of individual smaller structures and not a coherent circumstellar shell: "Its probable origin in the early nineteenth century .... makes formation of the outer ejecta by fragmentation of an expanding shell seem unlikely. Its morphology, high velocities and large velocity dispersion of individual structures, and the X-ray emission formed through shocks, support its creation in a more explosive event, during which several outer layers of the star's surface were ejected."

It appears from this that apart from the formation of the Homunculus there has been a period of large asymmetric mass ejections in the axis of the Homunculus, involving outer layers of the star. Weis (2012) estimates the combined mass of these ejecta to be at least $2-4 \mathrm{M}_{\odot}$, but such estimates are highly uncertain and easily could be as large as $10 \mathrm{M}_{\odot}$ or more. We present the amount of mass lost in the merger events in Tab. 2. The mass of the inner nitrogen enriched 
shell (the inner loop in fig. 5 ) comprises about $\sim 2.9 \mathrm{M}_{\odot}$, of the total ejected $\sim 22.2 \mathrm{M}_{\odot}$. In the other hydrodynamical simulations a similar fraction of the ejected mass (of about $15 \%-25 \%)$ is deposited in the nitrogen enriched skirt.

The large asymmetric mass ejection from the equatorial regions of the star (assuming the orbital plane of the original inner binary to coincide with the equatorial plane) is precisely what our merger model predicts. As a consequence, our model predicts that the current orbital plane is alinged with the equatorial plane of the Homunculus, and the symmetric lobes are roughtly aligned with the argument of periastron of the current Eta Carina binary.

\subsection{Composition of the outflow}

In Fig. 6 we notice that the inner loop (blue in the figure) in spatial vs velocity coordinates is enhanced in nitrogen by about a factor of 10 compared to the outer loop (red in fig. 6). The same is the case for carbon, but there the outer ring is composed of carbon poor material (with an abundance of $<0.0002$ ) and the inner structure is enhanced in carbon by more than an order of magnitude $(>0.003)$. A similar, but less extreme, bimodal distribution is observed in the oxygen content, but the range in abundance is much smaller.

The abundance differences across the remnant are due to the inspiraling process of the two stars. In the first violent passage mostly the outer layers are ejected, and upon each subsequent burst mass from the deeper layers of the primary star are ejected. Those deeper layers are more enhanced in CNO processed material.

Shortly after the merger, the star is bloated to about $120 \mathrm{R}_{\odot}$, and rapidly rotating (see $\S 2$ ). We did not follow the subsequent evolution of the merger product, because the Henyey stellar evolution code will be unable to follow the non-thermal evolution of the star properly. The current secondary star, which once was the outer tertiary star, will plunge through this envelope at its next pericenter passage, some $5.5 \mathrm{yr}$ after the merger event.

\section{ACKNOWLEDGMENTS}

It is a pleasure to thank Adrian Hamers, Tjarda Boekholt, Alex Rimoldi and Arnout van Gelderen for discussions, and the anonymous referee for grittycally reading the manuscript. This work was supported by the Netherlands Research Council NWO (grants \#643.200.503, \#639.073.803 and \#614.061.608) by the Netherlands Research School for Astronomy (NOVA) and by the National Science Foundation under Grant No. NSF PHY11-25915 Part of the numerical computations were carried out on the Little Green Machine at Leiden University.

\section{REFERENCES}

Aerts, C., Lamers, H. J. G. L. M., Molenberghs, G. 2004, A\&A , 418, 639

Damineli, A. 1996, ApJL, 460, L49

Damineli, A., Conti, P. S., Lopes, D. F. 1997, New Astron. $, 2,107$
Table 3. Reconstructed parameters for Eta Carinae. The first column identifies the parameter, and the subsequent columns give estimates for the conditions at birth, during the 1838 merger event and today. The latter are taken from the literature (Smith 2013). The parameters are the age of the primary star at the moment of the collision, the total mass of the inner binary, the mass lost in that phase of the evolution, and the mass of the outer component (later to become the secondary star). We further give the inner binary orbital parameters $\left(a_{\text {in }}\right.$ and $\left.e_{\text {in }}\right)$ and the outer elements $\left(a_{\text {out }}\right.$ and $\left.e_{\text {out }}\right)$. The last four parameters give the relative inclination of the two orbits, the mean anomaly of the outer orbit at the moment the merger, the kick velocity and its direction in the inner orbital plane.

\begin{tabular}{llll}
\hline & Phase A & Phase B & Phase C \\
\hline parameter & pre 1838 & 1838 & post 1838 \\
\hline$t$ & & $0.1-1.0 \mathrm{Myr}$ & $\lesssim 1 \mathrm{Myr}$ \\
$M_{\text {in }}+m_{\text {in }}$ & $120-150 \mathrm{M}_{\odot}$ & $114 \pm 12.2 \mathrm{M}_{\odot}$ & $90 \mathrm{M}_{\odot}$ \\
$d m_{\text {in }}$ & $22.4 \mathrm{M}_{\odot}$ & $23.7 \pm 12.2 \mathrm{M}_{\odot}$ & \\
$m_{\text {out }}$ & $30 \mathrm{M}_{\odot}$ & $30 \mathrm{M}_{\odot}$ & $30 \mathrm{M}_{\odot}$ \\
$a_{\text {in }}$ & $1-2 \mathrm{AU}$ & $0.36-0.8 \mathrm{AU}$ & \\
$e_{\text {in }}$ & $0-0.8$ & $0-0.96$ & \\
$a_{\text {out }}$ & $20-100 \mathrm{AU}$ & $20-100 \mathrm{AU}$ & $15.46 \mathrm{AU}$ \\
$e_{\text {out }}$ & $0.02-0.7$ & $0.02-0.7$ & 0.9 \\
$i$ & - & $91^{\circ} \pm 21^{\circ}$ & \\
mean anomaly & - & $0^{\circ} .061 \pm 0^{\circ} .035$ & \\
$v_{\text {kick }}$ & - & $53.1 \pm 7.7 \mathrm{~km} / \mathrm{s}$ & \\
$\theta$ & - & $180^{\circ} \pm 9^{\circ}$ & \\
\hline
\end{tabular}

Davidson, K., Humphreys, R. M. 1997, ARA\&A , 35, 1 de Vaucouleurs, G., Eggen, O. J. 1952, PASP , 64, 185 de Vries, N., Portegies Zwart, S., Figueira, J. 2014, MNRAS , 438, 1909

Eggleton, P. 2006, Evolutionary Processes in Binary and Multiple Stars, Evolutionary Processes in Binary and Multiple Stars, by Peter Eggleton, pp. . ISBN 0521855578. Cambridge, UK: Cambridge University Press, 2006.

Eggleton, P. P., Kiseleva, L. G., Hut, P. 1998, ApJ , 499, 853

Eggleton, P. P., Tokovinin, A. A. 2008, MNRAS , 389, 869

Eggleton, P. P., Tokovinin, A. A. 2010, VizieR Online Data Catalog, 738, 90869

Eisenstein, D. J., Hut, P. 1998, ApJ , 498, 137

Foley, R. J., Berger, E., Fox, O., Levesque, E. M., Challis, P. J., Ivans, I. I., Rhoads, J. E., Soderberg, A. M. 2011, ApJ , 732, 32

Ford, E. B., Kozinsky, B., Rasio, F. A. 2000, ApJ , 535, 385

Foreman-Mackey, D., Hogg, D. W., Lang, D., Goodman, J. 2013, PASP , 125, 306

Hamers, A. S., Perets, H. B., Portegies Zwart, S. F. 2015, ArXiv e-prints

Hansen, B. M. S. 2010, ApJ , 723, 285

Harpaz, A., Soker, N. 2009, New Astron. , 14, 539

Hastings, W. K. 1970, Biometrika, 57(1), 97

Hills, J. G. 1983, ApJ , 267, 322

Humphreys, R. M., Davidson, K. 1999, in J. A. Morse, R. M. Humphreys, A. Damineli (eds.), Eta Carinae at The Millennium, Vol. 179 of Astronomical Society of the 
Pacific Conference Series, 216

Humphreys, R. M., Martin, J. C. 2012, in K. Davidson, R. M. Humphreys (eds.), Astrophysics and Space Science Library, Vol. 384 of Astrophysics and Space Science Library, p. 1

Kashi, A., Frankowski, A., Soker, N. 2010, ApJL , 709, L11

Kashi, A., Soker, N. 2010, ApJ , 723, 602

Kato, T. 2003, A\&A , 399, 695

Kochanek, C. S., Szczygieł, D. M., Stanek, K. Z. 2012, ApJ , 758, 142

Kozai, Y. 1962, AJ , 67, 591

Kundt, W., Hillemanns, C. 2003, Chinese Journal of Astronomy and Astrophysics Supplement, 3, 349

Levesque, E. M., Stringfellow, G. S., Ginsburg, A. G., Bally, J., Keeney, B. A. 2014, AJ , 147, 23

Lidov, M. 1962, Planet. Space Sci., 9, 719

Livio, M., Pringle, J. E. 1998, MNRAS , 295, L59

Maeder, A. 1999, A\&A , 347, 185

Mardling, R. A. 2008, in S. J. Aarseth, C. A. Tout, R. A. Mardling (eds.), The Cambridge N-Body Lectures, Vol. 760 of Lecture Notes in Physics, Berlin Springer Verlag, 59

Mauerhan, J. C., Smith, N., Filippenko, A. V., Blanchard, K. B., Blanchard, P. K., Casper, C. F. E., Cenko, S. B., Clubb, K. I., Cohen, D. P., Fuller, K. L., Li, G. Z., Silverman, J. M. 2013, MNRAS , 430, 1801

Maza, J., Hamuy, M., Antezana, R., Gonzalez, L., Lopez, P., Silva, S., Folatelli, G., Iturra, D., Cartier, R., Forster, F., Marchi, S., Rojas, A., Pignata, G., Conuel, B., Reichart, D., Ivarsen, K., Haislip, J., Crain, A., Foster, D., Nysewander, M., Lacluyze, A. 2009, Central Bureau Electronic Telegrams, 1928, 1

Melendez, M. B., Gull, T. R., Bautista, M. A., Badnell, N. R. 2005, in American Astronomical Society Meeting Abstracts, Vol. 37 of Bulletin of the American Astronomical Society, \#114.11

Miroshnichenko, A. S., Pasechnik, A. V., Manset, N., Carciofi, A. C., Rivinius, T., Štefl, S., Gvaramadze, V. V., Ribeiro, J., Fernando, A., Garrel, T., Knapen, J. H., Buil, C., Heathcote, B., Pollmann, E., Mauclaire, B., Thizy, O., Martin, J., Zharikov, S. V., Okazaki, A. T., Gandet, T. L., Eversberg, T., Reinecke, N. 2013, ApJ , 766, 119

Morris, T., Podsiadlowski, P. 2006, MNRAS , 365, 2

Munari, U., Henden, A., Kiyota, S., Laney, D., Marang, F., Zwitter, T., Corradi, R. L. M., Desidera, S., Marrese, P. M., Giro, E., Boschi, F., Schwartz, M. B. 2002, A\&A , 389, L51

Nandez, J. L. A., Ivanova, N., Lombardi, Jr., J. C. 2014, ApJ , 786, 39

Owocki, S. P., Shaviv, N. J. 2012, in K. Davidson, R. M. Humphreys (eds.), Astrophysics and Space Science Library, Vol. 384 of Astrophysics and Space Science Library, 275

Parrent, J., Levitan, D., Howell, A., Thomas, R. C., Nugent, P., Sullivan, M., Kasliwal, M., Ofek, E. O., Quimby, R., Ben-Ami, S., Xu, D., Arcavi, I., Gal-Yam, A., Cenko, C. B., Li, W., Filippenko, A. V. 2011, The Astronomer's Telegram, 3510, 1

Pasquali, A., Nota, A., Langer, N., Schulte-Ladbeck, R. E., Clampin, M. 2000, AJ , 119, 1352

Paxton, B., Bildsten, L., Dotter, A., Herwig, F., Lesaffre,
P., Timmes, F. 2011, ApJS , 192, 3

Pelupessy, F. I., Jänes, J., Portegies Zwart, S. 2012, New Astron. , 17, 711

Pelupessy, F. I., van der Werf, P. P., Icke, V. 2004, A\&A , 422, 55

Pelupessy, F. I., van Elteren, A., de Vries, N., McMillan, S. L. W., Drost, N., Portegies Zwart, S. F. 2013, A\&A , 557, A84

Pijloo, J. T., Caputo, D. P., Portegies Zwart, S. F. 2012, MNRAS , 424, 2914

Portegies Zwart, S. 2013, MNRAS , 429, L45

Portegies Zwart, S., Boekholt, T. 2014, The Astrophysical Journal Letters, 785(1), L3

Portegies Zwart, S., McMillan, S. L. W., van Elteren, E., Pelupessy, I., de Vries, N. 2013, Computer Physics Communications, 183, 456

Rest, A., Prieto, J. L., Walborn, N. R., Smith, N., Bianco, F. B., Chornock, R., Welch, D. L., Howell, D. A., Huber, M. E., Foley, R. J., Fong, W., Sinnott, B., Bond, H. E., Smith, R. C., Toledo, I., Minniti, D., Mandel, K. 2012, Nat , 482, 375

Sana, H., de Koter, A., de Mink, S. E., Dunstall, P. R., Evans, C. J., Henault-Brunet, V., Maiz Apellaniz, J., Ramirez-Agudelo, O. H., Taylor, W. D., Walborn, N. R., Clark, J. S., Crowther, P. A., Herrero, A., Gieles, M., Langer, N., Lennon, D. J., Vink, J. S. 2012, ArXiv e-prints Sepinsky, J. F., Willems, B., Kalogera, V., Rasio, F. A. 2007, ApJ , 667, 1170

Smith, N. 2011, MNRAS , 415, 2020

Smith, N. 2013, MNRAS , 429, 2366

Smith, N., Frew, D. J. 2011, MNRAS , 415, 2009

Smith, N., Humphreys, R. M., Gehrz, R. D. 2001, PASP , 113,692

Smith, N., Li, W., Silverman, J. M., Ganeshalingam, M., Filippenko, A. V. 2011, MNRAS , 415, 773

Smith, N., Mauerhan, J. C., Cenko, S. B., Kasliwal, M. M., Silverman, J. M., Filippenko, A. V., Gal-Yam, A., Clubb, K. I., Graham, M. L., Leonard, D. C., Horst, J. C., Williams, G. G., Andrews, J. E., Kulkarni, S. R., Nugent, P., Sullivan, M., Maguire, K., Xu, D., Ben-Ami, S. 2015, ArXiv e-prints

Smith, N., Owocki, S. P. 2006, ApJL , 645, L45

Smith, N., Tombleson, R. 2015, MNRAS , 447, 598

Smith, N., Townsend, R. H. D. 2007, ApJ , 666, 967

Soker, N., Kashi, A. 2013, ApJL , 764, L6

Soker, N., Tylenda, R. 2003, ApJL , 582, L105

Springel, V. 2005, MNRAS , 364, 1105

Tauris, T. M., Takens, R. J. 1998, A\&A , 330, 1047

Thackeray, A. D. 1961, The Observatory, 81, 99

Tsebrenko, D., Soker, N. 2013, ApJL , 777, L35

Tylenda, R., Górny, S. K., Kamiński, T., Schmidt, M. 2014, ArXiv e-prints

Tylenda, R., Hajduk, M., Kamiński, T., Udalski, A., Soszyński, I., Szymański, M. K., Kubiak, M., Pietrzyński, G., Poleski, R., Wyrzykowski, Ł., Ulaczyk, K. 2011, A\&A , 528, A114

Tylenda, R., Soker, N. 2006, A\&A , 451, 223

van Boekel, R., Kervella, P., Schöller, M., Herbst, T., Brandner, W., de Koter, A., Waters, L. B. F. M., Hillier, D. J., Paresce, F., Lenzen, R., Lagrange, A.-M. 2003, A\&A , 410, L37

von Zeipel, H. 1924, MNRAS , 84, 665 
Walborn, N. R., Blanco, B. M., Thackeray, A. D. 1978, ApJ , 219, 498

Webbink, R. F. 1984, ApJ , 277, 355

Weigelt, G., Kraus, S. 2012, in K. Davidson, R. M. Humphreys (eds.), Astrophysics and Space Science Library, Vol. 384 of Astrophysics and Space Science Library, 129

Weis, K. 2012, in K. Davidson, R. M. Humphreys (eds.), Astrophysics and Space Science Library, Vol. 384 of Astrophysics and Space Science Library, 171 